\title{
Fauna helmintológica de ovinos provenientes da microrregião de Jaboticabal, estado de São Paulo, Brasil
}

\author{
Helminth fauna of sheep from the micro region of Jaboticabal, São Paulo State, Brazil
}

\author{
Willian Giquelin Maciel ${ }^{\mathrm{I}}$ Gustavo Felippelli ${ }^{\mathrm{I}}$ Welber Daniel Zanetti Lopes ${ }^{\mathrm{I}^{*}}$ \\ Weslen Fabricio Pires Teixeira ${ }^{\mathrm{I}}$ Breno Cayeiro Cruz ${ }^{\mathrm{I}}$ Thais Rabelo dos Santos ${ }^{\mathrm{I}}$ \\ Carolina Buzzulini ${ }^{\mathrm{I}}$ Flavia Favero ${ }^{\mathrm{I}}$ Lucas Costa Gomes ${ }^{\mathrm{I}}$ Gilson Pereira de Oliveira ${ }^{\mathrm{I}}$ \\ Alvimar José da Costa ${ }^{\mathrm{I}}$ Lucas Vinícius Shigaki de Matos ${ }^{\mathrm{I}}$
}

\section{RESUMO}

\begin{abstract}
Avaliou-se a prevalência e a contagem parasitária das diferentes espécies de helmintos de ovinos provenientes da microrregião de Jaboticabal, região Noroeste de São Paulo. Para tanto, foram utilizados 66 animais naturalmente infectados, de quatro a 36 meses de idade, criados em regime extensivo. Os resultados necroscópicos revelaram a presença de sete gêneros e 12 espécies, com a seguinte prevalência e a média de parasitismo: Haemonchus contortus: 100,0\% (2947,2); Trichostrongylus colubriformis: 90,9\% (3048,8); Cooperia curticei: 56,0\% (256,5); Oesophagostomum columbianum: 48,4\% (36,0); Cooperia punctata: $30,3 \%$ (94,5); Trichostrongylus axei: $22,7 \%(26,5)$; Strongyloides papillosus: 19,6\% (83,0); Haemonchus contortus (L4): 7,5\% (17,2); Cooperia pectinata: 10,6\% (12,9); Trichuris ovis: $10,6 \%(0,6)$; Cooperia spatulata $4,5 \%(0,3)$; Capillaria bovis: $4,5 \%(0,1)$. A carga parasitária média foi de 6.524,7 helmintos por animal. Haemonchus contortus (Adultos e L4) e Trichostrongylus colubriformis corresponderam a $45,4 \%$ e $46,7 \%$ da carga parasitária média total, respectivamente. Pode-se concluir que as duas espécies de helmintos mais abundantes e importantes da microrregião de Jaboticabal/São Paulo foram Trichostrongylus colubriformis e Haemonchus contortus, sendo que essas duas espécies perfizeram 92,1\% da distribuição percentual dos helmintos recolhidos de todos os animais. Tais resultados demonstram a importância em se realizar um monitoramento das contagens de ovos por grama de fezes (OPG) dos rebanhos desta região, quando o método FAMACHA for empregado em uma determinada propriedade, uma vez que este método de controle, geralmente, não permite diagnosticar os danos/sinais clínicos desencadeados nos animais pelo T. colubriformis, em função de essa espécie não possuir hábito de hematofagismo sobre os hospedeiros.
\end{abstract}

Palavras-chave: FAMACHA, nematódeos, prevalência, helmintos, ovinos.

\section{ABSTRACT}

This study evaluated the prevalence and counting parasitism of different species of helminths of sheep from the micro- region of Jaboticabal of São Paulo state. For this, 66 animals naturally infected, four to 36 months of age, raised in pasture, were selected. The results of necropsy revealed the presence of seven genera and 12 species with the following prevalence and mean count: Haemonchus contortus: 100.0\% (2947.2); Trichostrongylus colubriformis: $90.9 \%$ (3048.8); Cooperia curticei: $56.0 \%$ (256.5); Oesophagostomum columbianum: 48.4\% (36.0); Cooperia punctata: 30.3\% (94.5); Trichostrongylus axei: $22.7 \%$ (26.5); Strongyloides papillosus: $19.6 \%$ (83.0), Haemonchus contortus (L4): $7.5 \%$ (17.2), Cooperia pectinata: 10.6\% (12.9), Trichuris ovis: $10.6 \%$ (0.6); Cooperia spatulata 4.5\% (0.3); Capillaria bovis: $4.5 \%$ (0.1). The mean parasitism of helminthswas 6524.7 per animal. Haemonchus contortus (adults and L4) and Trichostrongylus colubriformis corresponded to $45.4 \%$ and $46.7 \%$ of the average worm burden totally, respectively. Based in the results obtained in this study, can be concluded that the two most abundant species of helminths and important, the micro-region of Jaboticabal are Trichostrongylus colubriformis and Haemonchus contortus, and these two species amounted to $92.1 \%$ of the distribution percentage of helminths collected from all animals. These results demonstrate the importance of conducting a counts of eggs per gram of feces (EPG) in the herds of this region when FAMACHA is used on a particular property, since this method control does not allow to diagnostic the damage/ clinical signs in animals infected by T. colubriformis, because this specie does not have hematophagism habit on animals.

Key words: FAMACHA, nematode, prevalence, helminths, sheep.

\section{INTRODUÇÃO}

As infecções por nematódeos gastrintestinais em ovinos determinam importantes perdas econômicas em decorrência da elevada mortalidade e morbidade. Atua de forma clínica e ou

ICentro de Pesquisas em Sanidade Animal (CPPAR), Faculdade de Ciências Agrárias e Veterinárias, Universidade Estadual Paulista “Júlio de Mesquita Filho” (UNESP), Via de acesso prof. Paulo Donatto Castellani, s/n, 14884-900, Jaboticabal, SP, Brasil. E-mail: wdzlopes@hotmail.com. *Autor para correspondência. 
subclínica em ovinos, prejudicando o desenvolvimento dos animais nas fases de cria e recria e diminuindo consequentemente a resistência destes às infecções desencadeadas por outros agentes parasitários como bactérias e vírus (VILELA et al., 2012).

De acordo com AMARANTE et al. (2004), para se obter maior sucesso no combate aos helmintos, é necessário realizar um controle embasado no conhecimento das espécies de nematódeos presentes nos animais da região, assim como a epidemiologia destes. Esses mesmos autores ressaltam ainda que o sistema de manejo zootécnico e sanitário, associado aos estudos epidemiológicos dos helmintos, podem diminuir a utilização deste tipo de medicamento nos animais.

Os trabalhos que enfatizam os indicadores de infecções helmínticas em ovinos, utilizando necropsia parasitológica no Brasil foram realizados por GONÇALVEZ (1974), SANTIAGO et al. (1976), VASCONCELOS et al. (1985), PINHEIRO et al. (1987), RAMOS et al. (2004). Considerando-se o elevado entrave que os nematódeos gastrintestinais podem representar na criação de ovinos, aliados ao fato de existirem poucos estudos sobre a fauna de helmintos nesta espécie animal, o presente trabalho teve como objetivo caracterizar a prevalência e a contagem parasitária das diferentes espécies de helmintos em ovinos, oriundos da microrregião de Jaboticabal, região Noroeste do Estado de São Paulo.

\section{MATERIAL E MÉTODOS}

Local e seleção dos animais

A pesquisa foi realizada de janeiro a dezembro 2012. Foram utilizados 66 ovinos pertencentes a 13 diferentes propriedades rurais da microrregião de Jaboticabal/SP (situada na região Noroeste do Estado de São Paulo, aproximadamente 350km da capital do estado), situadas nos seguintes municípios: Jaboticabal, Bebedouro, Matão, Viradouro, Pitangueiras, Ribeirão Preto e Pindorama.. Foram utilizados neste estudo ovinos machos e fêmeas, mestiços (Lanados e Deslanados), com idade entre quatro e 36 meses, criados em regime extensivo, naturalmente infectados por helmintos e que não eram criados em contato com outra espécie animal (bovino ou caprino). Apenas animais não tratados com qualquer anti-helmíntico nos 60 dias que antecederam esta pesquisa, e aqueles com as contagens de ovos (estrongilídeos) por grama de fezes (GORDON \& WHITLOCK, 1939), acima de 800 , foram selecionados. Vale frisar que, em nenhuma das propriedades de onde os animais foram obtidos, realizava-se métodos de controle alternativos (não químicos) contra helmintos. Em todas as propriedades de onde foram obtidos os animais, os ovinos recebiam tratamento anti-helmíntico a cada 40 ou 50 dias, ou antes desse intervalo, quando se observava sinal de edema submandibular nos animais.

Necropsias parasitológicas e identificação dos helmintos

Após seleção, todos os animais foram encaminhados ao setor de ovinos e caprinos do "Centro de Pesquisas em Sanidade Animal” (CPPAR/ FCAVJ/UNESP- Jaboticabal, SP). Decorrido o período de adaptação, todos os animais foram eutanasiados, conforme os procedimentos éticos descritos no Guidelines on Euthanasia of American Veterinary Medical Association AVMA - (2007). Na sequência, todos os tratos gastrintestinais dos ovinos foram removidos, e os vários segmentos anatômicos (abomaso, intestino delgado e intestino grosso), isolados e separados por ligaduras duplas. Após a colheita, o conteúdo foi armazenado em potes plásticos acrescidos de formol a 10\% préaquecido. A mucosa do abomaso de cada animal foi submetida à digestão com solução pepsina/ clorídrica previamente aquecida a aproximadamente $37^{\circ} \mathrm{C}$ de quatro a seis horas (WOOD et al., 1995). Além disso, todos os pulmões e fígados também foram dissecados e inspecionados visualmente, com o objetivo de determinar a presença e o número de helmintos (adultos e larvas), possivelmente presentes nestes órgãos (WOOD et al., 1995). Após armazenamento, foi retirada uma alíquota de $10 \%$ (pós-homogeneização) de cada conteúdo previamente armazenado em formol. Os helmintos foram colhidos usando estereomicrospio, e as suas identificações genéricas e específicas, utilizando um microscópio óptico (ampliação de 100-400 x), de acordo com os critérios taxonomicos descritos por COSTA (1982) e UENO \& GONÇALVES (1998).

\section{RESULTADOS}

Os resultados necroscópicos, presentes na tabela 1, revelaram a presença de 430.635,0 helmintos, pertencentes a sete gêneros e 12 espécies, com a seguinte prevalência e a média de parasitismo: Haemonchus contortus: 100,0\% (2947,2); Trichostrongylus colubriformis: $90,9 \%$ (3048,8); Cooperia curticei: 56,0\% (256,5); Oesophagostomum columbianum: 48,4\% (36,0); Cooperia punctata: 30,3\% (94,5); Trichostrongylus axei: $22,7 \%$ (26,5); Strongyloides papillosus: 19,6\% (83,0); Haemonchus contortus (L4): 7,5\% (17,2); Cooperia pectinata: $10,6 \%(12,9)$; Trichuris

Ciência Rural, v.44, n.3, mar, 2014. 
ovis: 10,6\% (0,6); Cooperia spatulata 4,5\% (0,3); Capillaria bovis: 4,5\% (0,1).

A carga parasitária média foi de 6.524,7 helmintos por animal. Por meio da identificação dos helmintos colhidos nas necropsias, observouse que Haemonchus contortus (Adultos e L4) e Trichostrongylus colubriformis corresponderam a $45,4 \%$ e $46,7 \%$ da carga parasitária média total, respectivamente (Tabela 1). Apesar da pequena diferença percentual demonstrada pela espécie $\mathbf{T}$. colubriformis em comparação ao $\boldsymbol{H}$. contortus (Adultos e L4), é importante frisar que os 66 animais (100\% de prevalência) necropsiados encontravamse parasitados por adultos $\boldsymbol{H}$. contortus, enquanto que, em 60 ovinos, foram encontrados adultos de $\boldsymbol{T}$. colubriformis (90,9\% de prevalência). Os demais $7,8 \%$ da carga parasitária média diagnosticada é representada por: C. curticei (3,9\%), C. punctata (1,4\%), S. papillosus $(1,2 \%)$, O. columbianum (0,5\%), T. axei $(0,4 \%)$, C. pectinata $(0,2 \%), C$. spatulata $(0,01 \%)$, T. ovis $(0,01 \%)$ e C. bovis (0,001\%).

A distribuição percentual dos casos de infecçãoregistrada de acordo com onúmero de espécies de helmintos presentes em um mesmo hospedeiro está inserida na tabela 2. Pela análise da referida tabela, observa-se que $86,3 \%$ dos ovinos estavam infectados por duas a seis espécies de helmintos. Dentre estes, 46,9\% estavam parasitados por apenas duas ou três espécies. Apenas 9,0\% dos hospedeiros mostraram-se parasitados com sete ou oito diferentes espécies de helmintos em seu trato digestivo. Em nenhum animal foi possível observar mais de oito espécies de helmintos em conjunto (Tabela 2). Fato interessante que se deve relatar é que, de um modo geral, os animais que estavam parasitados com sete ou oito espécies de helmintos, continham uma carga parasitária média de $\boldsymbol{H}$. contortus e $\boldsymbol{T}$. colubriformis relativamente menor, quando comparados aos demais ovinos diagnosticados com uma a seis espécies de nematódeos em conjunto (Tabela 3).

A observação visual dos fígados e pulmões neste estudo, durante a necropsia dos 66 ovinos, não revelou a presença de nenhum parasito.

\section{DISCUSSÃO}

RAMOS et al. (2004), necropsiando ovinos traçadores no planalto catarinense, evidenciaram que H. contortus e T. colubriformis foram as espécies de helmintos mais encontradas no abomaso e intestino delgado, respectivamente. Resultados semelhantes a estes também foram encontrados anteriormente por GONÇALVES(1974) e PINHEIRO et al. (1987) no Rio Grande do Sul, e próximo a mesma região de onde este estudo foi conduzido por VASCONCELOS et al. (1985). Nesse caso, evidencia-se que, decorridos mais de 30 anos, não houve alteração significativa da

Tabela 1 - Prevalência, contagem média e amplitude de variação de helmintos em ovinos da região microrregião de Jaboticabal, Estado de São Paulo.

\begin{tabular}{|c|c|c|c|c|}
\hline \multirow[b]{2}{*}{ Espécies de helmintos } & \multirow[b]{2}{*}{ Prevalência } & \multicolumn{2}{|c|}{-------------Contagem da infecção helmíntica-------------- } & \multirow[b]{2}{*}{$\begin{array}{l}\text { Amplitude de variação } \\
\text { da infecção helmíntica }\end{array}$} \\
\hline & & $\begin{array}{l}\text { Contagem média de helmintos } \\
\text { entre ovinos necropsiados }\end{array}$ & $\begin{array}{l}\text { Distribuição percentual } \\
\text { de helmintos (\%) }\end{array}$ & \\
\hline Haemonchus contortus (adultos) & $100,0 \%$ & 2947,2 & 45,2 & $03-16.745$ \\
\hline Haemonchus contortus (L4) & $7,5 \%$ & 17,3 & 0,3 & $00-1.050$ \\
\hline Cooperia punctata & $30,3 \%$ & 94,5 & 1,4 & $00-1.695$ \\
\hline Cooperia curticei & $56,0 \%$ & 256,5 & 3,9 & $00-4.293$ \\
\hline Cooperia pectinata & $10,6 \%$ & 13,0 & 0,2 & $00-273$ \\
\hline Cooperia spatulata & $4,5 \%$ & 0,3 & 0,0 & $00-16$ \\
\hline Trichostrongylus axei & $22,7 \%$ & 26,6 & 0,4 & $00-1.239$ \\
\hline Trichostrongylus colubriformis & $90,9 \%$ & 3048,8 & 46,7 & $00-32.923$ \\
\hline Trichostrongylus logispicuralis & $1,5 \%$ & 0,7 & 0,0 & $00-46$ \\
\hline Strongyloides papillosus & $19,6 \%$ & 83,1 & 1,3 & $00-3240$ \\
\hline Oesophagostomum columbianum & $48,4 \%$ & 36,0 & 0,6 & $00-440$ \\
\hline Trichuris ovis & $10,6 \%$ & 0,7 & 0,0 & $00-20$ \\
\hline Capillaria bovis & $4,5 \%$ & 0,1 & 0,0 & $00-03$ \\
\hline \multicolumn{5}{|l|}{ Moniezia } \\
\hline Total & - & 6524,77 & 100,000 & - \\
\hline
\end{tabular}

Ciência Rural, v.44, n.3, mar, 2014. 
fauna helmintológica dos ovinos da região, apesar de possíveis alterações no manejo, emprego de lactonas macrocíclicas, ou, ainda, das supostas alterações climáticas globais durante o referido período.

DOMINGUES et al. (2013) relatam que as principais espécies de helmintos que acometem os ovinos, dependendo da região, são o Haemonchus contortus e Trichostrongylus colubriformis. Esses autores enfatizam, ainda, que $\boldsymbol{H}$. contortus é o nematódeo gastrintestinal mais patogênico de ovinos, responsável pela enfermidade denominada de hemoncose, sendo a anemia e dispepsia (seguida de hipoproteinemia e edemas) os principais sinais clínicos dessa infecção parasitária. Já o T. colubriformis, responsável pela enfermidade denominada de tricostrongilose, causa uma gastrenterite parasitária com secreção de muco, podendo ainda ocorrer hemorragia, com exsudação de líquidos e desequilíbrio eletrolítico, resultando em um quadro clínico de hipoproteinemia/edemas e diarreia (AMARANTE et al., 2004). Na maioria dos casos, T. colubriformis é considerado um helminto que pode ocasionar problemas secundários ao $\boldsymbol{H}$. contortus em animais. Entretanto, os resultados de prevalência deste estudo demonstram que $\boldsymbol{T}$. colubriformis pode ser tão importante quanto $\boldsymbol{H}$. contortus nesta região e que, muito provavelmente, $\boldsymbol{T}$. colubriformis esteja ocasionando danos aos animais, mais imperceptíveis aos produtores de ovinos quando comparados aos prejuízos desencadeados por $\boldsymbol{H}$. contortus. Os resultados encontrados por CARDIA et al. (2011) reforçam tal inferência. Esses autores avaliaram a resposta imunológica e o desempenho de crescimento de ovinos Santa Inês, experimentalmente infectados por T. colubriformis, e concluíram que os animais infectados com o referido helminto apresentaram atrofia das vilosidades intestinais e menor conversão alimentar, o que, por sua vez, acabou desencadeando uma retardo no crescimento desses ovinos, provocado pelos helmintos adultos de T. colubriformis presentes na mucosa intestinal. MARTINS FILHO \& MENEZES (2001) e RAMOS et al. (2004) encontraram em ovinos baixo parasitismo e prevalência por Trichuris e Oesophagostomum, assim como no presente estudo.

Prevalência de 19,6\% foi encontrada para S. papillosus no presente estudo. Resultados superiores foram encontrados em ovinos e caprinos por MARTINS FILHO \& MENEZES (2001). Apesar de esses autores não relatarem a idade dos animais em seus respectivos estudos, de acordo com COSTA et al. (1979), a baixa prevalência desta espécie de helminto, pode estar associada com a faixa etária dos animais envolvidos no estudo, uma vez que, a partir dos cinco meses, ruminantes começam a demonstrar resistência contra essa espécie e, neste caso, a maior parte dos ovinos tinham idade superior a cinco meses.

Cooperia punctata, C. pectinata, $C$. spatulata, Trichostrongylus axei, T. logispicuralis, Trichuris ovis e Capillaria bovis estavam presentes nos ovinos, entretanto, com baixo nível de infecção e prevalência nos animais, evidenciando-se pouca importância no contexto epidemiológico da região onde o estudo foi conduzido.

Em relação à não detecção de helmintos nos pulmões e fígado dos 66 animais necropsiados neste estudo, fica claro a baixa incidência e prevalência destas espécies que têm estes locais como habitat, nos rebanhos de ovinos da região onde o estudo foi realizado. Baixo ou ausência de parasitismo nestes segmentos anatômicos também foi relatado em ovinos por outros pesquisadores (RAMOS et al., 2004).

Tabela 2 - Distribuição percentual, média/desvio padrão e amplitude de variação da infecção helmíntica dos casos de coinfecções helmínticas nos ovinos da microrregião de Jaboticabal, Estado de São Paulo.

\begin{tabular}{|c|c|c|c|c|c|c|}
\hline \multirow{2}{*}{$\begin{array}{l}\text { № de Espécies encontradas no } \\
\text { mesmo animal }\end{array}$} & \multirow{2}{*}{$\begin{array}{l}\text { Número de animais } \\
\text { infectados } \\
3\end{array}$} & \multirow{2}{*}{$\begin{array}{c}\text { Porcentagem } \\
4,5\end{array}$} & \multicolumn{3}{|c|}{--------Média e Desvio Padrão-------- } & \multirow{2}{*}{$\begin{array}{c}\text { Amplitude de variação } \\
\text { da infecção helmíntica }\end{array}$} \\
\hline & & & 305,6 & \pm & 1686,8 & \\
\hline 2 & 14 & 21,2 & 122,1 & \pm & 420,0 & $00-3.434$ \\
\hline 3 & 17 & 25,7 & 659,4 & \pm & 2847,0 & $00-32.923$ \\
\hline 4 & 5 & 7,5 & 248,6 & \pm & 789,7 & $00-4.805$ \\
\hline 5 & 12 & 18,1 & 273,7 & \pm & 1143,5 & $00-11.231$ \\
\hline 6 & 9 & 13,6 & 393,1 & \pm & 1209,5 & $00-8.379$ \\
\hline 7 & 4 & 6,0 & $1.800,0$ & \pm & 5288,2 & $00-26.170$ \\
\hline 8 & 2 & 3,0 & $1.632,0$ & \pm & 3998,6 & $00-15.998$ \\
\hline Total & 66 & 100 & - & - & - & - \\
\hline
\end{tabular}


Tabela 3 - Contagem média de infecção por Haemonchus conctortus e Trichostrongylus colubriformis em ovinos com coinfecção helmíntica.

\begin{tabular}{lccc}
\hline No de Espécies encontradas no mesmo animal & Quantidade & $\begin{array}{l}\text { Contagem média da infecção por } \\
\text { H. contortus }\end{array}$ & $\begin{array}{l}\text { Contagem média da infecção por } \\
\text { T. colubriformis }\end{array}$ \\
\hline 1 a 6 & 60 & 3100,8 & 3148,8 \\
7 a 8 & 6 & 1518,0 & 911,6 \\
Total & 66 & - & - \\
\hline
\end{tabular}

No que diz respeito ao número de espécies encontradas no mesmo ovino (51,5\% dos animais parasitados com uma a três espécimes), estes resultados podem ser justificados em função dos achados descritos por GIUDICI et al. (1999). Esses autores, em seu estudo, verificaram que a diversidade de espécies de nematódeos em ovinos é maior quando eles compartilham pastagens com bovinos, fato este que não aconteceu com os animais do presente estudo. Por outro lado, LOPES et al. (2013) ressaltam ainda que as dosificações frequentes (tratamentos supressivos) aumentam as chances de prevalência de uma única espécie parasitária, geralmente a mais resistente as bases farmacêuticas utilizadas em um determinado rebanho. O FAMACHA, um método adotado para o controle de helmintos, foi inicialmente descoberto e utilizado em ovinos, entretanto, recentes estudos comprovam bons resultados dessa medida no controle da verminose, tanto de ovinos quanto de caprinos (MOLENTO et al., 2004; VILELA et al., 2012). É importante frisar que tal ferramenta é utilizada na prática de acordo com a coloração da mucosa ocular inferior dos animais (de vermelho robusto a branco), de modo que sua indicação acaba sendo especificamente para o controle de espécies hematófagas, principalmente $\boldsymbol{H}$. contortus, que possui elevada prevalência nos rebanhos, e um considerável poder de hematofagismo sobre os animais. Os resultados de prevalência e distribuição percentual das principais espécies de helmintos diagnosticadas na região onde o estudo foi conduzido $(\boldsymbol{H}$. contortus e $\boldsymbol{T}$. colubriformis) demonstram a importância em se realizar um monitoramento constante das contagens de ovos por grama de fezes (OPG) dos rebanhos. Nesse sentido, quando o método FAMACHA for empregado em uma determinada propriedade, uma vez que o parasitismo por $\boldsymbol{T}$. colubriformis na região é no mínimo equivalente ao por $\boldsymbol{H}$. contortus, aliado ao fato de que este método de controle, geralmente, não permite diagnosticar os danos/sinais clínicos desencadeados nos animais pelo T. colubriformis, uma vez que esta espécie não possui hábito de hematofagismo sobre os hospedeiros.

\section{CONCLUSÃO}

Com base nos resultados encontrados no presente trabalho, pode-se concluir que as duas espécies de helmintos mais abundantes e importantes da microrregião de Jaboticabal/São Paulo, nas 66 necropsias parasitológicas, foram Trichostrongylus colubriformis (46,7\%) e Haemonchus contortus (45,4\%), sendo que essas duas espécies perfizeram 92,1\% da distribuição percentual dos helmintos recolhidos de todos os animais. Tais resultados demonstram a importância em se realizar um monitoramento das contagens de ovos por grama de fezes (OPG) dos rebanhos desta região, quando o método FAMACHA for empregado em uma determinada propriedade, uma vez que este método de controle, geralmente, não permite diagnosticar os danos/sinais clínicos desencadeados nos animais pelo T. colubriformis, em função de esta espécie não possuir hábito de hematofagismo sobre os hospedeiros.

\section{COMITÊ DE ÉTICA E BIOSEGURANÇA}

Este trabalho foi submetido e aprovado pelo comitê de ética, sob processo 012J2/2011.

\section{REFERÊNCIAS}

AMARANTE, A.F.T. et al. Resistance of santa ines, suffolk and ile de France lambs to naturally acquired gastrointestinal nematode infections. Vet Parasitol, v.120, p.91-106, 2004. Disponível em: <http:/www.sciencedirect.com/science/article/pii/ S0304401703005041>. Acesso em: 02 maio 2013. doi: dx.doi. org/10.1016/j.vetpar.2003.12.004.

CARDIA, D.F.F. et al. Immune response and performance of growing Santa Ines lambs to artificial Trichostrongylus colubriformis infections. Vet Parasitol, v.182, p.248258, 2011. Disponível em: <http:/www.ncbi.nlm.nih.gov/ pubmed/21641720>. Acesso em: 10 ago. 2013. doi: 10.1016/j. vetpar.2011.05.017.

COSTA, A.J. et al. Helmintos parasitos de bezerros do município de Uruana - Goiás, Brasil. Arq Esc Vet, v.31, p.33-36, 1979.

COSTA, A.J. Diagnóstico laboratorial em Parasitologia. I. Helmintologia. Jaboticabal-SP: FCAV-UNESP, 1982. 89p. 
DOMINGUES, L.F. et al. In vitro and in vivo evaluation of the activity of pineapple (Ananas comosus) on Haemonchus contortus in Santa Inês sheep. Vet Parasitol, v.193, p.263-270, 2013. Disponível em: <http://www.sciencedirect.com/science/ article/pii/S0304401713002434>. Acesso em: 10 ago. 2013. doi: dx.doi.org/10.1016/j.vetpar.2013.04.031.

GIUDICI, C. et al. Changes in gastro-intestinal helminth species diversity in lambs under mixed grazing on irrigated pastures in the tropics (French West Indies). Vet Res, v.30, p.573581, 1999. Disponível em: <http://www.ncbi.nlm.nih.gov/ pubmed/10596405>. Acesso em: 10 ago. 2013.

GONÇALVES, P.C. Epidemiologia da helmintose ovina em Guaíba (RS). 1974. 41f. Dissertação (Mestrado em Doenças Parasitárias) - Universidade Federal do Rio Grande do Sul, RS.

GORDON, H.M.; WHITLOCK, H.V. A new techinique for counting nematode eggs in sheep faeces. J Counn Sci Ind Res Aust, v.12, p.50-52, 1939.

LOPES, W.D.Z. et al. Persistent efficacy of 3.5\% doramectin compared to $3.15 \%$ ivermectin against gastrointestinal nematodes in experimentally-infected cattle in Brazil. Res Vet Sci, v.94, p.290-294, 2013. Disponível em: <http://www.ncbi.nlm.nih.gov/ pubmed/23102648>. Acesso em: 02 maio 2013. doi: 10.1016/j. rvsc.2012.09.022.

MARTINS FILHO, E.; MENEZES, R.C.A.A. Parasitos gastrintestinais em caprinos (Capra hircus) de uma criação extensiva na microregião de Curimataú, Estado da Paraíba, Brasil. Rev Bras Par Vet, v.10, p.41-44, 2001. Disponível em: <http:// www.bdpa.cnptia.embrapa.br/busca>. Acesso em: 02 maio 2013.

MOLENTO, M.B. et al. Método FAMACHA como parâmetro clínico individual de infecção por Haemonchus contortus em pequenos ruminantes. Ciênc Rural, v.34, p.1139-1145, 2004. Disponível em: <http://www.scielo.br/pdf/cr/v34n4/a27v34n4. pdf $>$. Acesso em: 02 maio 2013.
NOTE for Guidelines on Euthanasia. (American Veterinary Medical Association) 36p, 2007. Disponível em: <http://grants. nih.gov/grants/olaw/Euthanasia2007.pdf $>$. Acesso em: 02 maio 2013

PINHEIRO, A.C. et al. Epidemiologia da helmintose ovina em Bagé (RS-Brasil). Bagé-RS: Centro Nacional de Pesquisa em Ovinos, EMPRESA BRASILEIRA DE PESQUISA AGROPECUÁRIA, 1987. p.263-267. (Coletânea das pesquisas: Medicina Veterinária e Parasitologia. Bagé).

RAMOS, C.I. et al. Epidemiologia das helmintoses gastrintestinais de ovinos no Planalto Caratinense. Cienc. Rural, v.34, p.18891895, 2004. Disponível em: <http://www.scielo.br/scielo. php?pid=S0103-84782004000600034\&script $=$ sci_arttext $>$. Acesso em: 18 nov 2013.doi: dx.doi.org/10.1590/S010384782004000600034

UENO, H.; GONÇALVES, P.C. Manual para diagnóstico das helmintoses de ruminantes. 4.ed. Japão: JICA, 1998. 166p.

VASCONCELOS, O.T. et al. Parâmetros parasitológicos, coprométricos e necroscópicos em ovinos do município de Catanduva, Estado de São Paulo. Ars Vet, v.1, p.89-101, 1985.

VILELA, V.L.R.et al. FAMACHA method as an auxiliary strategy control of gastrointestinal helminthiasis of dairy goats under semiarid conditions of Brazil northeastern. Vet. Parasitol, v.190, p.281-284, 2012. Disponível em: <http://www.ncbi.nlm.nih.gov/ pubmed/22726386>. Acesso em: 02 maio 2013. doi: 10.1016/j. vetpar.2012.05.024.

WOOD, I.B. et al. World Association for the Advancement of Veterinary Parasitology (W.A.A.V.P.): second edition of guidelines for evaluating the efficacy of anthelmintics in ruminants (bovine, ovine, caprine). Vet Parasitol, v.58, p.181-213, 1995. Disponível em: <http://www.ncbi.nlm.nih.gov/pubmed/7571325>. Acesso em: 02 maio 2013. 